\title{
Theranosties
}

Editorial

2012; 2(2):125-126. doi: 10.7150/thno.4129

\section{Protease Activity: Meeting Its Theranostic Potential}

\section{Seulki Lee ${ }^{1 凶}$ and Kwangmeyung Kim ${ }^{2 \bowtie}$}

1. Laboratory of Molecular Imaging and Nanomedicine (LOMIN), National Institute of Biomedical Imaging and Bioengineering, National Institutes of Health, USA

2. Center for Theragnosis, Biomedical Research Institute, Korea Institute of Science and Technology, Korea

$\triangle$ Corresponding author: seulki.lee@nih.gov, kim@kist.re.kr

(C) Ivyspring International Publisher. This is an open-access article distributed under the terms of the Creative Commons License (http://creativecommons.org/ licenses/by-nc-nd/3.0/). Reproduction is permitted for personal, noncommercial use, provided that the article is in whole, unmodified, and properly cited.

Received: 2012.01.21; Accepted: 2012.01.21; Published: 2012.02.01

\begin{abstract}
This themed issue provides up-to-date review and research articles covering the theranostic applications in the combined fields of protease research, diagnostics and drug development.
\end{abstract}

Key words: Biomarkers, diagnosis, drug development, molecular imaging, proteases, theranostics

Proteases, which are also known as proteolytic enzymes, are important signaling proteins that play crucial roles in a number of pathological processes, such as cancer, inflammatory and cardiovascular diseases and neurological disorders [1]. Proteases were previously considered enzymes that only catalyze protein degradation by hydrolysis of peptide bonds. Nowadays, proteases are recognized as exceptionally important molecules that are engaged in numerous vital life processes. Because certain states of protease activity can affect, lead to or signal specific diseases, it is not surprising that proteases have been actively investigated as potential therapeutic and imaging targets. For instance, various novel protease inhibitors and protease-targeted prodrugs are under active clinical investigations and some of which are expected to enter the market in the next few years [2-4]. Since certain types of proteases can be used as biomarkers and/or prognostic markers, different types of diagnostics measuring proteases' activities have been widely developed [5]. In addition, recent technological developments in molecular imaging have allowed detecting and imaging protease activities in living organisms using different imaging modalities. In this special issue of Theranostics, we would like to share ideas and opinions on why pro- tease-targeted imaging and therapy is attractive in the drug development and diagnosis field through up-to-date review and research articles.

This special issue contains 10 review and research articles. The paper by Kim and Kim [6] presents the design of quantum dot-based molecular probes that can sense protease activities via fluorescence and bioluminescence resonance energy transfer systems. These promising protease sensors are expected to identify and contribute to the understanding of the specific role of proteases in particular biological processes and have great potential as high throughput drug screening tools for screening novel protease inhibitors as therapeutic drugs. In nature, proteolytic degradation often induces or sensitizes the controlled self-assemblies of certain proteins. Inspired by these unique phenomena, various self-assembled supramolecular nanostructures built up by enzymatic processes have been studied. The exciting article by Chen and Liang [7] focuses on the concept and application of these enzyme-catalyzed or -regulated nanostructures for theranostics. In another review article, Chen et al. [8] introduces how molecular imaging techniques can contribute to understanding the signaling cascades involved in cell death by targeting important kinases and proteases. The paper by Choi et al. [5] 
introduces protease-targeted sensing and imaging applications, focuses on the strategies to develop sophisticated protease-sensitive prodrugs targeting various diseases and elucidates the importance of their therapeutic applications in the field of drug development.

A number of research articles introduce new theranostic materials or systems targeting specific proteases in vivo, in cells and in clinical samples. Yhee et al. [9] report fluorogenic peptides targeting two popular proteases, cathepsin B and matrix metalloproteinases (MMPs), and their applications in cancer theranostics. Jang and Choi [10] show a similar substrate using an MMP fluorogenic peptide that can successfully turn into an theranostic agent when combined with photodynamic agents and gold nanorods. They demonstrate both imaging and therapeutic applicability of this MMP-activatable photosensitizing system in cellular models. Clinical translation of in vitro MMP diagnostics was also reported. Ryu et al. [11] exhibit an MMP diagnostic kit designed by immobilization of polymer-MMP probe conjugates and exemplify its use in monitoring early stages of osteoarthritis and acute inflammatory conditions of the knee joint by measuring MMP activities in the synovial fluids from patients. Two papers [12, 13] describe methods of real-time monitoring of apoptosis in vivo and in cells by imaging the activity of the apoptotic protease, caspase-3, using bioluminescent and fluorescent proteins that are sensitive to caspase-3. Since the most effective anticancer therapeutics rely on apoptosis, developing new techniques that enable real-time imaging of apoptotic processes will improve the understanding of drug-induced apoptosis and assist in the screening of apoptosis-related novel drug candidates. Lastly, the exciting paper by Habibollahi et al. [14] report a highly clinically translatable technique by demonstrating how imaging protease biomarkers can improves the detection of esophageal adenocarcinoma in orthotopic small animal models when combined with a dual-channel fluorescent endoscopic system.

The field of protease research is far-reaching ranging fundamental biology to drug development and to molecular imaging. Currently, one of the hot topics of research is the identification and validation of target proteases for drug development and diagnosis. Understanding the complex protease signaling in various disease conditions should be established to make these unique molecules useful in our lives. For successful real applications, multidisciplinary research, like the combination of biological knowledge, engineering technologies, and clinical tools, is needed. Although a few examples were provided in this themed issue, there is no doubt that theranostics will play the role of locomotive, translating protease-related applications into the clinic. We thank all the authors for contributing their papers and hope the topics covered in this issue will provide new information to readers of Theranostics.

\section{Conflict of Interest}

The authors have declared that no conflict of interest exists.

\section{References}

1. Lopez-Otin C and Bond JS. Proteases: Multifunctional enzymes in life and disease. J Biol Chem. 2008; 283: 30433-7.

2. Turk B. Targeting Proteases: Successes, failures and future prospects. Nat Rev Drug Discov. 2006; 5: 785-99.

3. Rautio J, Kumpulainen H, Heimbach T, et al. Prodrugs: Design and clinical applications. Nat Rev Drug Discov. 2008; 7: 255-70.

4. Choi KY, Swierczewska M, Lee S, et al. Protease-activated drug development. Theranostics. 2012; 2: 156-178.

5. Zhu L, Xie J, Swierczewska M, et al. Real-time video imaging of protease expression in vivo. Theranostics. 2011; 1: 18-27.

6. Kim GB and Kim Y-P. Analysis of protease activity using quantum dots and resonance energy transfer. Theranostics. 2012; 2: 127-138

7. Chen $Y$ and Liang G. Enzymatic self-assembly of nanostructures for theranostics. Theranostics. 2012; 2: 139-147.

8. Chen HH, Yuan H, Josephson L, et al. Theranostic imaging of the kinases and proteases that modulate cell death and survival. Theranostics. 2012; 2: 148-155.

9. Yhee JY, Kim SA, Koo H, et al. Optical imaging of cancer-related proteases using near-infrared fluorescence matrix metalloproteinase-sensitive and cathepsin b-sensitive probes. Theranostics. 2012; 2: 179-189.

10. Jang B and Choi Y. Photosensitizer-conjugated gold nanorods for enzyme-activatable fluorescence imaging and photodynamic therapy. Theranostics. 2012; 2: 190-197.

11. Ryu JH, Lee A, Huh MS, et al. Measurement of mmp activity in synovial fluid in cases of osteoarthritis and acute inflammatory conditions of the knee joints using a fluorogenic peptide probe-immobilized diagnostic kit. Theranostics. 2012; 2: 198-206.

12. Ozaki M, Haga $S$, and Ozawa T. In vivo monitoring of liver damage using caspase-3 probe. Theranostics. 2012; 2: 207-214.

13. Savitsky AP, Rusanov AL, Zherdeva VV, et al. Flim-fret imaging of caspase- 3 activity in live cells using pair of red fluorescent proteins. Theranostics. 2012; 2: 215-226.

14. Habibollahi P, Figueiredo J-L, Heidari P, et al. Optical imaging with a cathepsin $\mathrm{b}$ activated probe for the enhanced detection of esophageal adenocarcinoma by dual channel fluorescent upper gi endoscopy. Theranostics. 2012; 2: 227-234. 